\title{
The Effect of Obesity and Diabetes on Intermediate to High Grade Prostate Cancer Patients Treated with Radical Prostatectomy
}

\author{
Emma H. Ramahi ${ }^{*}$, Katherine C. Ansley ${ }^{1 *}$, Gregory P. Swanson ${ }^{1,2,3}$, Fei Du ${ }^{1,4}$, Joseph W. Basler ${ }^{1,3}$ \\ ${ }^{1}$ The University of Texas Health Science Center at San Antonio, San Antonio, USA \\ ${ }^{2}$ Department of Radiation Oncology, San Antonio, USA \\ ${ }^{3}$ Department of Urology, San Antonio, USA \\ ${ }^{4}$ Department of Epidemiology and Biostatistics, San Antonio, USA \\ Email: swansong@uthscsa.edu
}

Received December 14, 2011; revised January 20, 2012; accepted January 30, 2012

\begin{abstract}
Aims: The relationships between obesity, diabetes and prostate cancer are unclear. A retrospective study was performed to determine the effects of body mass index (BMI) and diabetes on patients with intermediate to high grade prostate cancer treated with radical prostatectomy. Methods: We reviewed 582 patients with Gleason score $\geq 7$ non-metastatic prostate cancer treated with radical prostatectomy. Patients were stratified by BMI. End points were biochemical failure free survival (BFFS), overall survival (OS), and cancer specific survival (CSS). Results: Mean pre-treatment PSA decreased with increasing BMI $(12.5,7.6,7.8$ and $5.3 \mathrm{ng} / \mathrm{mL}$ with BMI $<25,25-30,30-35$, and $>35$, respectively; $\mathrm{p}<$ 0.001). There was no significant difference in BFFS, OS or CSS between diabetic and non-diabetic patients. After adjusting for potential confounders (age, Gleason score and pre-treatment PSA), patients with higher BMI experienced biochemical failure more often with hazard ratios $1.87(1.15,3.04 ; \mathrm{p}=0.01), 2.23(1.39,3.56 ; \mathrm{p}<0.001)$, and $2.5(1.22$, $5.12 ; \mathrm{p}=0.01)$ for BMI $25-30,30-35$ and $>35$, respectively. However, for overall mortality the adjusted hazard ratio was $0.39(0.18,0.82 ; \mathrm{p}=0.01)$ for overweight patients (BMI $25-30)$ compared to patients with a BMI in the normal range. Patients with a BMI of $30-35$ and $>35$ had increased rates of positive margins than those with a BMI of $25-30$ or $<25(41.4 \%$ and $45.5 \%$ versus $28.9 \%$ and $33.3 \%$, respectively; $\mathrm{p}=0.02)$. Patients with higher BMI than 25 had higher biochemical recurrence rate ( 25 - 30 HR 2; 30 - 35 HR 1.97 and >35 2.04) on multivariate analysis, margin positivity alone was not a significant factor. Conclusions: Patients with increasing BMI tend to have a lower PSA at diagnosis but are more likely to have biochemical failure after radical prostatectomy. In our cohort, this was not due to the increased incidence of positive margins. Having diabetes had no effect.
\end{abstract}

Keywords: Obesity; Diabetes Mellitus; Prostatectomy; Prostatic Neoplasms; Therapy; Treatment Outcomes

\section{Introduction}

Prostate cancer and obesity are important causes of morbidity and mortality in the United States. Prostate cancer is the most commonly diagnosed non-cutaneous cancer in American men, with approximately 220,000 cases identified each year [1]. It is estimated that prostate cancer will represent $28 \%$ of new male cancer diagnoses and will be the second leading cause of cancer death in men in 2010 [1].

Approximately one third of American men are clinically obese, and the prevalence of obesity has risen 6 to 7 percent each decade since the 1980s [2]. Obesity is a well-known risk factor for a variety of chronic conditions including hypertension, high cholesterol, stroke, heart

*E. H. Ramahi and K. C. Ansley contributed equally to this work. disease, and certain cancers such as colon, kidney, and esophageal [3]. As obesity has grown more prevalent, the incidence of type 2 diabetes mellitus has also increased, and obesity increases the risk of death from diabetes up to 9 times [4].

Faced with the increased incidence of prostate cancer and obesity-related health issues, several investigators have focused on how an increased body mass index (BMI) and the presence of diabetes impact the incidence, diagnosis, and response to treatment for prostate cancer. Several studies show that obesity affects prostate cancer mortality rather than incidence $[5,6]$. For example, in the RTOG 85 - 31 trial of patients with locally advanced prostate cancer undergoing radiation, it was found that 5year prostate cancer specific mortality for men with a $\mathrm{BMI}<25$ was $6.5 \%$ compared with $13.1 \%$ and $12.2 \%$ in 
men with $\mathrm{BMI} \geq 25-30$ and $\mathrm{BMI} \geq 30$ respectively [7]. Therefore a greater baseline BMI was independently associated with risk of death from prostate cancer. More recent research, however, have not supported these findings, so the association remains uncertain $[8,9]$.

In addition, there is some evidence that shows type 2 diabetes is significantly linked with a decreased risk of developing prostate cancer [10-12], but few studies have evaluated the impact of diabetes on prostate cancer mortality. One recent study evaluated 112 diabetic metformin users and 98 diabetic non-metformin users treated with radical prostatectomy and demonstrated that diabetics, regardless of metformin use, faced a 55\% increase in risk of biochemical recurrence [13]; this was even though there is some evidence that metformin may have some antineoplastic effects [14].

Given the uncertainty regarding the effects of obesity, diabetes and diabetes treatment on prostate cancer outcome, we reviewed the outcome of our patients with Gleason 7 - 10 cancer that were treated with radical prostatectomy for these associations.

\section{Materials and Methods}

With institutional review board approval, we reviewed patients in the South Texas Veteran's Healthcare System Tumor Registry diagnosed with prostate cancer between January 1, 1998 and December 31, 2008. We entered patients with Gleason scores greater than or equal to 7 on biopsy or on radical prostatectomy pathology into a database. 123 patients had a biopsy Gleason score $<7$, but were found to have Gleason 7 - 10 disease on radical prostatectomy pathology. Statistical analysis was performed both with and without the inclusion of these patients, and their inclusion did not lead to significant differences in outcome measure comparison.

Patient information including age at diagnosis, BMI at the time of treatment initiation, race, presence or absence of diabetes, the HbA1c value recorded closest to the time of diagnosis, and choice of treatment for diabetes, classified as either non-pharmacologic, metformin, other oral medication or insulin were all obtained from the medical record. Patients were coded to have diabetes when the diagnosis was made and recorded in the medical record by the patient's primary physician prior to initiation of treatment for prostate cancer. Also recorded were the PSA immediately prior to diagnosis, information from the original biopsy report including the Gleason score as determined by the original attending pathologist, and whether positive cores came from one or both sides of the prostate. Information from the surgical pathology report was also recorded including surgical Gleason score as determined by the original attending pathologist, the presence of disease in seminal vesicles (SV) or lymph nodes (LN), the presence of extracapsular extension (ECE), and the presence of positive margins. Patients were excluded from analysis if they had metastatic disease at the time of diagnosis as identified on bone scan, computed tomography scan or magnetic resonance imaging. Patients were also excluded from analysis if their BMI was not recorded in the medical record or if they were lost to follow up before post-treatment PSA values could be obtained.

Biochemical failure was defined by the American Urological Association Prostate Cancer Guideline Panel's definition of a PSA of $0.2 \mathrm{ng} / \mathrm{mL}$ or greater followed by a confirmatory PSA value $0.2 \mathrm{ng} / \mathrm{mL}$ or greater [15]. Patients receiving a second modality as salvage treatment were also considered to have failed on the date salvage treatment was initiated, even if the above criteria had not been met.

Patients were stratified into groups based on BMI into the following categories: normal (BMI $<25)$, overweight (BMI 25 - 29.9), obese (BMI 30 - 35) and morbidly obese $($ BMI > 35). Additionally, patients were stratified based on the presence or absence of the diagnosis of diabetes at the time of treatment initiation, whether or not their diabetes was controlled as evidence by $\mathrm{HbA} 1 \mathrm{c} \leq 7$ or $>7$, and by the treatment for diabetes: non-pharmacologic, metformin, other oral agents, or insulin.

\section{Statistical Methods}

Continuously distributed data were summarized with the sample size, mean and standard deviation (SD) and categorical data were described with counts and percentages. Data were grouped by BMI category and diabetic status. Groups were contrasted on continuously distributed outcomes with Kruskal-Wallis tests. The significance of associations between categorical outcomes and BMI category, diabetic status, and treatment were assessed with Pearson's chi-square test. BMI categories were contrasted with regard to biochemical free and overall survival with proportional hazards models and Kaplan Meier survival curves and associated log rank tests. Hazard ratios and their $95 \%$ confidence intervals are reported. Hazard ratios were adjusted for potential confounders: age, Gleason score, pre-treatment PSA, the presence of diabetes and surgical pathology. All statistical testing was 2 -sided with a significance level of $5 \%$. SAS Version 9.2 for Windows (SAS Institute, Cary, North Carolina) was used throughout.

\section{Results}

Mean follow up was $5.1 \pm 2.8$ years. A total of 582 patients with Gleason score $\geq 7$ were analyzed by BMI and diabetes category. Not surprisingly, there was a significant trend of increasing incidence of diabetes with in- 
creasing BMI. Specifically, for men with BMI > 35, more than $50 \%$ were diabetic, while the incidence was one third or less in those with BMI $\leq 35$. There was a significant decrease in PSA at diagnosis as BMI increased; at the extremes, men with BMI $<25$ had an average PSA of $12.5 \mathrm{ng} / \mathrm{ml}$, while those with BMI $>35 \mathrm{had}$ an average PSA of $5.3 \mathrm{ng} / \mathrm{ml}$. There was no significant difference for Gleason score or the presence of bilateral disease found on biopsy (Table 1). There was also no significant difference between the BMI groups in terms of RP surgical pathology features such as positive lymph nodes, positive seminal vesicles and extracapsular extension. There were, however, significantly higher rates of positive margins in patients with a BMI of $30-35$ and $>$ 35 when compared to patients with a BMI of $<25$ and 25 - 30 (Table 2).

BMI did not significantly affect unadjusted 5-year BFFS or CSS (Table 3). Kaplan-Meier logistical regression analysis likewise showed no significant difference in unadjusted BFFS (Figure 1). Neither the presence of diabetes, the degree of control as evidenced by HbAlc at diagnosis, nor the treatment regimen for diabetes had a significant effect on 5 year BFFS, OS or CSS.

Patients using metformin had a $61.3 \% 5 \mathrm{yr}$ BFFS, while for the non users (not using metformin) it was $71 \%$ $(p=0.09)$. There was no difference in CSS or OS.

After controlling for age, Gleason score, pre-treatment PSA, margin, SV, and node positivity, patients with increasing BMI above 25 were found to be at significantly increasing risk of biochemical failure with hazard ratios $2(1.21,3.33 ; \mathrm{p}=0.007), 1.97(1.21,3.21 ; \mathrm{p}=0.006)$, and $2.04(0.98,4.26 ; \mathrm{p}=0.06)$ for BMI $25-30,30-35$ and $>35$, respectively (Table 4). Positive margins alone did not explain this with no significant difference in hazard rations for the different BMI groups. (Table 4).
Patients with the lowest $(<25)$ BMI were found to have significantly lower unadjusted 5 -year OS $(86.3 \%$ compared to $93.6 \%, 94.6 \%$ and $93.2 \%$ for patients with BMI of $<25,25-29.9,30-35$ and $>35$, respectively $(p=0.05)$ (Table 3). Even after controlling for age, Gleason score, pre-treatment PSA and surgical pathology, moderately overweight patients with a BMI of $25-30$ and $30-35$ had a lower overall mortality than those with a normal BMI $(<25)$ as evidenced by a hazard ratio of $0.38(0.18$, $0.79 ; \mathrm{p}=0.01)$ and $0.48(0.23,1.0 ; \mathrm{p}=0.05)$. Those with a BMI $>35$ were nonsignificantly higher $(p=0.92)$ with a HR of $1.06(0.37,3.04)$ However, Kaplan-Meier estimates did not show a significant difference in unadjusted OS among the BMI groups.

\section{Discussion}

We were able to demonstrate an inverse relationship between BMI and PSA at diagnosis, supporting the multiple groups who have observed that men with prostate cancer and an increased BMI have a lower PSA [16-18] than thinner men with similar appearing cancers. An artificially low PSA in an obese man potentially could delay a prostate biopsy recommendation and increase risk for a higher-grade cancer [19]. One recent hypothesis for the low PSA in obese men faults hemodilution as a result of increased plasma volume [20-22]. In our cohort, patients are usually referred to urology for any elevation of the PSA by a large number of providers, but there could be some unknown selection factor that results in obese men being referred earlier (with a lower PSA). In obese men diagnosed with prostate cancer, Davies et al found that they are less likely to receive surgical treatment [23]. There are several possible reasons why a physician and their obese patient may decide against pursuing surgery

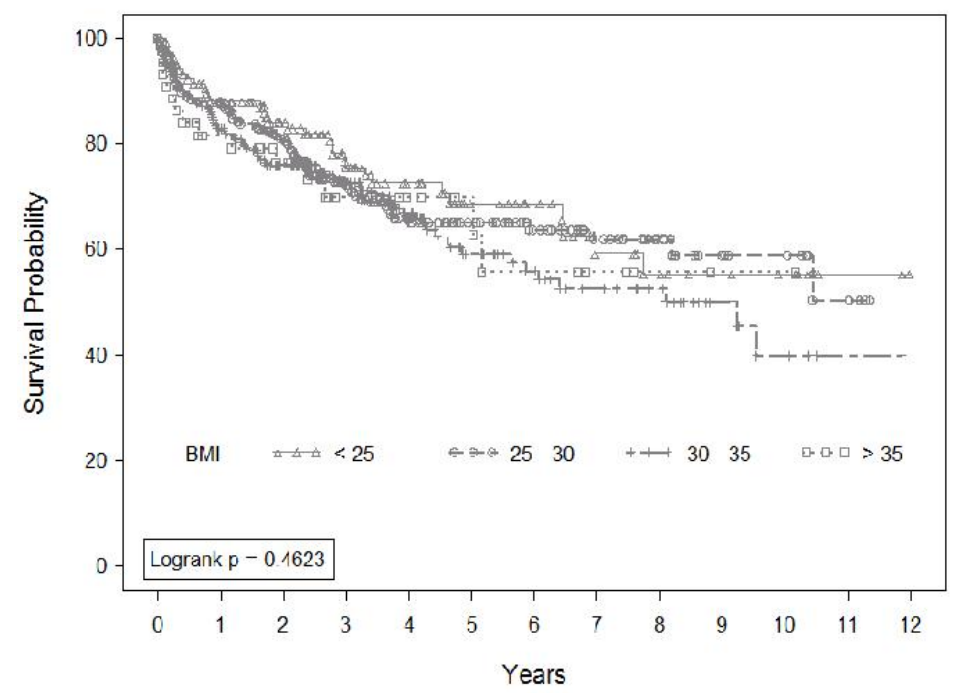

Figure 1. Unadjusted biochemical failure free survival by body mass index. 
Table 1. Pretreatment characteristics for patients undergoing radical prostatectomy for Gleason 7 - 10 prostate cancer.

\begin{tabular}{|c|c|c|c|c|c|c|}
\hline BMI: & $<25$ & $25-30$ & $30-35$ & $>35$ & Total & p-value ${ }^{1}$ \\
\hline Age & & & & & & $0.15^{1}$ \\
\hline $\mathrm{N}$ & 117 & 218 & 203 & 44 & 582 & \\
\hline Mean (SD) & $62.4(6.9)$ & $63.6(6.9)$ & $62.6(6.7)$ & $60.6(6.7)$ & $62.8(6.8)$ & \\
\hline Median (Q1, Q3) & $62.2(57.8,66.7)$ & $62.9(58.9,67.9)$ & $61.9(58.6,67.4)$ & $61.6(56.1,65)$ & $62.6(58.4,67.3)$ & \\
\hline Min, Max & $44.04,79$ & $46.56,88.1$ & $43.73,78.6$ & $36.7,71.8$ & $36.7,88.1$ & \\
\hline Race, n (\%) & & & & & & $0.46^{2}$ \\
\hline Unkown & $10(8.5)$ & $37(17)$ & $32(15.8)$ & $6(13.6)$ & $85(14.6)$ & \\
\hline White & $96(82.1)$ & $154(70.6)$ & $150(73.9)$ & $34(77.3)$ & $434(74.6)$ & \\
\hline Black & $10(8.5)$ & $24(11)$ & $16(7.9)$ & $4(9.1)$ & $54(9.3)$ & \\
\hline Other & $1(0.9)$ & $3(1.4)$ & $5(2.5)$ & $0(0)$ & $9(1.5)$ & \\
\hline Total & 117 & 218 & 203 & 44 & 582 & \\
\hline PSA at diagnosis & & & & & & $<0.001^{1}$ \\
\hline $\mathrm{N}$ & 111 & 214 & 194 & 40 & 559 & \\
\hline Mean (SD) & $12.5(23)$ & $7.6(8.8)$ & $7.8(7.2)$ & $5.3(2.2)$ & $8.5(12.5)$ & \\
\hline Median (Q1, Q3) & $6.7(5.1,11.3)$ & $5.5(4.2,8.3)$ & $5.4(4.2,8.4)$ & $4.9(4.2,6.6)$ & $5.6(4.3,8.6)$ & \\
\hline Min, Max & $0.74,221.9$ & $1.01,99.6$ & $0.52,48.1$ & $1.17,12.7$ & $0.52,221.9$ & \\
\hline Gleason score, n (\%) & & & & & & $0.55^{2}$ \\
\hline 7 & $57(60)$ & $119(70)$ & $108(67.9)$ & $21(60)$ & $305(66.4)$ & \\
\hline 8 & $21(22.1)$ & $24(14.1)$ & $30(18.9)$ & $8(22.9)$ & $83(18.1)$ & \\
\hline $9-10$ & $17(17.9)$ & $27(15.9)$ & $21(13.2)$ & $6(17.1)$ & $71(15.5)$ & \\
\hline Total & 95 & 170 & 159 & 35 & 459 & \\
\hline Bilateral disease, $\mathrm{n}(\%)$ & & & & & & $0.97^{2}$ \\
\hline Unilateral & $61(53)$ & $117(54.2)$ & $103(52.3)$ & $24(55.8)$ & $305(53.4)$ & \\
\hline Bilateral & $54(47)$ & $99(45.8)$ & $94(47.7)$ & $19(44.2)$ & $266(46.6)$ & \\
\hline Total & 115 & 216 & 197 & 43 & 571 & \\
\hline Diabetes, n (\%) & & & & & & $<0.001^{2}$ \\
\hline No & $104(88.9)$ & $170(78)$ & $136(67)$ & $21(47.7)$ & $431(74.1)$ & \\
\hline Yes & $13(11.1)$ & $48(22)$ & $67(33)$ & $23(52.3)$ & $151(25.9)$ & \\
\hline Total & 117 & 218 & 203 & 44 & 582 & \\
\hline $\mathrm{HbA1c}$ & & & & & & $0.58^{1}$ \\
\hline $\mathrm{N}$ & 12 & 47 & 66 & 23 & 148 & \\
\hline Mean (SD) & $6.7(1.5)$ & $6.7(1.3)$ & $6.9(1.6)$ & $6.9(0.9)$ & $6.8(1.4)$ & \\
\hline Median (Q1, Q3) & $6.1(5.8,7)$ & $6.5(5.8,7.2)$ & $6.5(5.9,7.5)$ & $6.6(6.3,7.5)$ & $6.5(5.9,7.4)$ & \\
\hline Min, Max & $5.6,10$ & $4.9,10.2$ & $5,12.4$ & $5.6,8.9$ & $4.9,12.4$ & \\
\hline Diabetes treatment, $\mathrm{n}(\%)$ & & & & & & $<0.001^{2}$ \\
\hline Metformin Use & $3(2.6)$ & $21(9.6)$ & $36(17.7)$ & $15(34.1)$ & $75(12.9)$ & \\
\hline Insulin & $2(1.7)$ & $8(3.7)$ & $4(2)$ & $1(2.3)$ & $15(2.6)$ & \\
\hline Other & $4(3.4)$ & $7(3.2)$ & $12(5.9)$ & $3(6.8)$ & $26(4.5)$ & \\
\hline No treatment & $108(92.3)$ & $182(83.5)$ & $151(74.4)$ & $25(56.8)$ & $466(80.1)$ & \\
\hline Total & 117 & 218 & 203 & 44 & 582 & \\
\hline
\end{tabular}

${ }^{1}$ Kruskal-wallis Test; ${ }^{2}$ Pearson's Chi-square Test. 
Table 2. Surgical pathology by body mass index in patients undergoing radical prostatectomy for Gleason 7 - 10 prostate cancer.

\begin{tabular}{|c|c|c|c|c|c|c|}
\hline & & & BMI & & & \\
\hline Surgical Pathology & $<25$ & $25-30$ & $30-35$ & $>35$ & Total & p-value ${ }^{1}$ \\
\hline$+\mathrm{LN}, \mathrm{N}(\%)$ & & & & & & $0.75^{2}$ \\
\hline No & $112(95.7)$ & $212(97.2)$ & $198(97.5)$ & $42(95.5)$ & $564(96.9)$ & \\
\hline Yes & $5(4.3)$ & $6(2.8)$ & $5(2.5)$ & $2(4.5)$ & $18(3.1)$ & \\
\hline Total & 117 & 218 & 203 & 44 & 582 & \\
\hline$+\mathrm{SV}, \mathrm{N}(\%)$ & & & & & & $0.39^{2}$ \\
\hline No & $104(88.9)$ & $193(88.5)$ & $176(86.7)$ & $35(79.5)$ & $508(87.3)$ & \\
\hline Yes & $13(11.1)$ & $25(11.5)$ & $27(13.3)$ & $9(20.5)$ & $74(12.7)$ & \\
\hline Total & 117 & 218 & 203 & 44 & 582 & \\
\hline + ECE, N (\%) & & & & & & $0.35^{2}$ \\
\hline No & $87(74.4)$ & $176(80.7)$ & $155(76.4)$ & $31(70.5)$ & $449(77.1)$ & \\
\hline Yes & $30(25.6)$ & $42(19.3)$ & $48(23.6)$ & $13(29.5)$ & $133(22.9)$ & \\
\hline Total & 117 & 218 & 203 & 44 & 582 & \\
\hline + Margins, N (\%) & & & & & & $0.02^{2}$ \\
\hline No & $78(66.7)$ & $155(71.1)$ & $119(58.6)$ & $24(54.5)$ & $376(64.6)$ & \\
\hline Yes & $39(33.3)$ & $63(28.9)$ & $84(41.4)$ & $20(45.5)$ & $206(35.4)$ & \\
\hline Total & 117 & 218 & 203 & 44 & 582 & \\
\hline
\end{tabular}

$\mathrm{BMI}=$ body mass index; $\mathrm{LN}=$ lymph nodes; $\mathrm{SV}=$ seminal vesicles; $\mathrm{ECE}=$ extracapsular extension; ${ }^{1}$ Kruskal-wallis Test $;{ }^{2}$ Pearson's Chi-square Test.

Table 3. 5-year survival outcomes by body mass index, presence, control and treatment of diabetes.

\begin{tabular}{|c|c|c|c|c|c|c|}
\hline & 5-year BFFS \% & p-value ${ }^{1}$ & 5 -year OS \% & p-value ${ }^{1}$ & 5-year CSS \% & p-value \\
\hline \multicolumn{7}{|c|}{ BMI } \\
\hline$<25$ & $75.2 \%$ & 0.46 & $86.3 \%$ & 0.05 & $100 \%$ & 0.54 \\
\hline $25-29.9$ & $69.3 \%$ & & $93.6 \%$ & & $98.6 \%$ & \\
\hline $30-35$ & $67 \%$ & & $94.6 \%$ & & $99 \%$ & \\
\hline$>35$ & $72.7 \%$ & & $93.2 \%$ & & $97.7 \%$ & \\
\hline \multicolumn{7}{|l|}{ Diabetes } \\
\hline No & $71.4 \%$ & 0.13 & $91.9 \%$ & 0.81 & $98.6 \%$ & 0.78 \\
\hline Yes & $65.6 \%$ & & $93.4 \%$ & & $99.3 \%$ & \\
\hline \multicolumn{7}{|l|}{$\mathrm{HbA1C}$} \\
\hline$\leq 7$ & $65.4 \%$ & 0.74 & $95.2 \%$ & 0.15 & $100 \%$ & 0.12 \\
\hline$>7$ & $68.2 \%$ & & $88.6 \%$ & & $97.7 \%$ & \\
\hline \multicolumn{7}{|c|}{ Diabetes treatment } \\
\hline Metformin use & $61.3 \%$ & 0.38 & $96 \%$ & 0.08 & $98.7 \%$ & 0.76 \\
\hline Insulin & $80 \%$ & & $80 \%$ & & $100 \%$ & \\
\hline Other & $65.4 \%$ & & $88.5 \%$ & & $100 \%$ & \\
\hline
\end{tabular}

${ }^{1}$ Kruskal-wallis Test.

as the primary therapeutic modality. Primarily, they tend to have less favorable outcomes than do thinner men, and there are several reasons possible reasons for this. Jaya- chandran et al. postulate that these inferior outcomes are due to the increased technical difficulty of the surgery in heavier patients [24]. This is supported by our findings of 
Table 4. Multivariate analysis for biochemical failure risk by body mass index.

\begin{tabular}{|c|c|c|c|c|}
\hline \multirow[b]{2}{*}{ BMI } & \multicolumn{2}{|c|}{$\begin{array}{l}\text { Control for Age, Gleason Score, } \\
\text { Pre-treatment PSA, Mar+, SV+ } \\
\text { and LN+ }\end{array}$} & \multicolumn{2}{|c|}{ Control for Mar+ } \\
\hline & HR (95\% CI) & $\mathbf{P}$ & HR $(95 \%$ CI $)$ & $\mathbf{P}$ \\
\hline$<25$ & 1.0 (reference) & - & 1.0 (reference) & - \\
\hline $25-30$ & $2(1.21,3.33)$ & 0.007 & $1.18(0.78,1.79)$ & 0.43 \\
\hline $30-35$ & $1.97(1.21,3.21)$ & 0.006 & $1.23(0.81,1.85)$ & 0.33 \\
\hline$>35$ & $2.04(0.98,4.26)$ & 0.06 & $1.14(0.61,2.14)$ & 0.67 \\
\hline
\end{tabular}

Age is continuous variable.

increased positive margins with increased BMI despite no significant difference in Gleason score, the rate of positive lymph nodes, seminal vesicles or extracapsular extension. These data support the finding that with larger patients, there is an increase in positive margins. Although we saw an increased risk of biochemical failure with increasing BMI, this was not explained by the finding of positive margins (Table 4). In fact, when corrected for known prognostic factors, obese patients still had a significantly higher failure rate. (Table 4).

While our data suggest these increasing rates of biochemical failure do not translate into decreased overall or cancer specific survival with our length of follow up, at the minimum these patients will be subjected more often to the morbidity of salvage treatments such as radiation therapy, hormone therapy, or chemotherapy. Unlike the RTOG 85 - 31 trial, we were unable to show worse CSS in patients with increased BMI. This is in agreement with the findings of two recent studies [8,9]. These studies have either shown no difference [8] or a trend toward decreasing OS with increasing BMI [9]. Kane et al analyzed patients in the Cancer of the Prostate Strategic Urologic Research Endeavor (CaPSURE) registry and found that overweight and obese patients with prostate cancer tended to have more medical comorbidities such as diabetes and hypertension. Kane et al. also hypothesized that overweight and obese men may have an increased diagnosis of low risk prostate cancer due to more frequent doctor's visits for their other co-morbid conditions [18]. Our data showed increasing rates of diabetes as BMI increased, so close medical follow up for this and other potential chronic medical condition may explain our finding of decreased mortality in the overweight BMI group (BMI 25 - 30) even after controlling for potential confounders such as age, Gleason score, pre-treatment PSA and the presence of diabetes. We also sought to explore the effect of diabetes and its treatment on 5-year OS, BFFS and CSS. Interestingly, some studies suggest diabetics may have a decreased risk of developing prostate cancer, which is thought to be due to multiple hormonal factors [25]. This raises the question as to whether those with cancer might fare better than non-diabetic patients. Unfortunately, Patel showed that diabetes, regardless of metformin use, resulted in a $55 \%$ increase in risk of biochemical failure [13]. However, we were unable to demonstrate any significant difference in outcome between diabetics and non-diabetics. In addition, even though Metformin is thought to exert both anti-tumor and anti-proliferative effects [26] and has been associated with decreased total cancer risk in epidemiologic studies, our patients did not appear to benefit from its use. If anything, in our patients, metformin users have a higher biochemical recurrence rate. Study limitations include those inherent to any retrospective analysis. Selection of treatment was at the discretion of the original treating physician. Also, our single HbAlc level at diagnosis is only a snapshot of diabetes control, so a detailed analysis of disease outcome based on that parameter is not possible. Additionally, a mean follow up of 5.1 years is still too short to make definitive statements regarding the ultimate development of metastatic disease and prostate cancer mortality. Further follow up will make this more clear.

\section{Conclusion}

Previous studies have raised the concern that increased BMI and diabetes adversely affect prostate cancer outcomes. As the average BMI and the incidence of diabetes continue to increase, these issues will affect more and more men. Our data suggest that although overweight and obese men tend to be younger and with a lower PSA at diagnosis, they are more likely to have co-morbid diagnoses such as diabetes. Most importantly, obese men are more likely to have positive surgical margins, which may contribute to the increased rate of biochemical recurrence after radical prostatectomy. Our data show no clear relationship between BMI and prostate cancer specific survival. There is uncertainty in the current literature as to the exact effect of obesity and diabetes on prostate cancer. Large scale prospective randomized controlled trials would be required to resolve these issues. Ultimately, the molecular mechanisms linking obesity, diabetes, and prostate cancer are multifactorial. In the meantime, as the morbidity and mortality associated with obesity and diabetes are well established, we should continue to encourage weight loss, increased physical activity, and glycemic control in our patients with prostate cancer.

\section{Acknowledgements}

The authors would like to thank Joel Michalek, PhD of the University of Texas Health Science Center at San Antonio Department of Epidemiology and Biostatistics for his assistance in editing this manuscript. 


\section{REFERENCES}

[1] A. Jemal, R. Siegel, J. Xu and E. Ward, "Cancer Statistics, 2010," Cancer Journal for Clinicians, Vol. 60, No. 5, 2010, pp. 277-300. doi:10.3322/caac.20073

[2] K. M. Flegal, M. D. Carroll, C. L. Ogden and L. R. Curtin, "Prevalence and Trends in Obesity among US Adults, 1999-2008," Journal of the American Medical Association, Vol. 303, No. 3, 2010, pp. 235-241.

doi:10.1001/jama.2009.2014

[3] E. E. Calle, C. Rodriguez, K. Walker-Thurmond and M. J. Thun, "Overweight, Obesity, and Mortality from Cancer in a Prospectively Studied Cohort of US Adults," The New England Journal of Medicine, Vol. 348, No. 17, 2003, pp. 1625-1638. doi:10.1056/NEJMoa021423

[4] R. G. Rogers, R. A. Hummer and P. M. Krueger, "The Effect of Obesity on Overall, Circulatory Disease- and Diabetes-Specific Mortality," Journal of Biosocial Science, Vol. 35, No. 1, 2003, pp. 107-129. doi:10.1017/S002193200300107X

[5] A. R. Kristal and Z. Gong, "Obesity and Prostate Cancer Mortality," Future Oncology, Vol. 3, No. 5, 2007, pp. 557-567. doi:10.2217/14796694.3.5.557

[6] P. Dimitropoulou, R. M. Martin, E. L. Turner, et al., "Association of Obesity with Prostate Cancer: A Case-Control Study within the Population-Based PSA Testing Phase of the Protect Study," British Journal of Cancer, Vol. 104, No. 5, 2011, pp. 875-881. doi:10.1038/sj.bjc. 6606066

[7] J. A. Efstathiou, K. Bae, W. U. Shipley, et al., "Obesity and Mortality in Men with Locally Advanced Prostate Cancer: Analysis of RTOG 85-31," Cancer, Vol. 110, No. 12, 2007, pp. 2691-2699. doi:10.1002/cncr.23093

[8] B. J. Davies, M. C. Smaldone, N. Sadetsky, et al., "The Impact of Obesity on Overall and Cancer Specific Survival in Men with Prostate Cancer," Journal of Urology, Vol. 182, No. 1, 2009, pp. 112-117. doi:10.1016/j.juro.2009.02.118

[9] J. Pfitzenmaier, M. Pritsch, A. Haferkamp, et al., "Is the Body Mass Index a Predictor of Adverse Outcome in Prostate Cancer after Radical Prostatectomy in a MidEuropean Study Population?" British Journal of Urology International, Vol. 103, No. 7, 2009, pp. 877-882. doi:10.1111/j.1464-410X.2008.08149.x

[10] S. Bonovas, V. Peponis and K. Filioussi, "Diabetes Mellitus as a Risk Factor for Primary Open-Angle Glaucoma: A Meta-Analysis," Diabetic Medicine, Vol. 21, No. 6, 2004, pp. 609-614. doi:10.1111/j.1464-5491.2004.01173.x

[11] J. S. Kasper and E. Giovannucci, "A Meta-Analysis of Diabetes Mellitus and the Risk of Prostate Cancer," Cancer Epidemiology, Biomarkers \& Prevention, Vol. 15, No. 11, 2006, pp. 2056-2062. doi:10.1158/1055-9965.EPI-06-0410

[12] E. A. Atchison, G. Gridley, J. D. Carreon, et al., "Risk of Cancer in a Large Cohort of US Veterans with Diabetes," International Journal of Cancer, Vol. 128, No. 3, 2011, pp. 635-643. doi:10.1002/ijc.25362

[13] T. Patel, G. Hruby, K. Badani, et al., "Clinical Outcomes after Radical Prostatectomy in Diabetic Patients Treated with Metformin," Urology, Vol. 76, No. 5, 2010, pp. 1240-1244. doi:10.1016/j.urology.2010.03.059

[14] J. M. Evans, L. A. Donnelly, A. M. Emslie-Smith, et al., "Metformin and Reduced Risk of Cancer in Diabetic Patients," British Medical Journal, Vol. 330, No. 7503, 2005, pp. 1304-1305. doi:10.1136/bmj.38415.708634.F7

[15] M. S. Cookson, G. Aus, A. L. Burnett, et al., "Variation in the Definition of Biochemical Recurrence in Patients Treated for Localized Prostate Cancer: The American Urological Association Prostate Guidelines for Localized Prostate Cancer Update Panel Report and Recommendations for a Standard in the Reporting of Surgical Outcomes," Journal of Urology, Vol. 177, No. 2, 2007, pp. 540-545. doi:10.1016/j.juro.2006.10.097

[16] L. L. Banez, L. Sun, B. J. Trock, et al., "Body Mass Index and Prostate Specific Antigen as Predictors of Adverse Pathology and Biochemical Recurrence after Prostatectomy," Journal of Urology, Vol. 182, No. 2, 2009, pp. 491-496. doi:10.1016/j.juro.2009.04.007

[17] J. Baillargeon, B. H. Pollock, A. R. Kristal, et al., "The Association of Body Mass Index and Prostate-Specific Antigen in a Population-Based Study," Cancer, Vol. 103, No. 5, 2005, pp. 1092-1095. doi:10.1002/cncr.20856

[18] C. J. Kane, W. W. Bassett, N. Sadetsky, et al., "Obesity and Prostate Cancer Clinical Risk Factors at Presentation: Data from CaPSURE," Journal of Urology, Vol. 173, No. 3, 2005, pp. 732-736. doi:10.1097/01.ju.0000152408.25738.23

[19] N. Parekh, Y. Lin, R. S. Dipaola, et al., "Obesity and Prostate Cancer Detection: Insights from Three National Surveys," American Journal of Medicine, Vol. 123, No. 9, 2010, pp. 829-835. doi:10.1016/j.amjmed.2010.05.011

[20] L. L. Banez, R. J. Hamilton, A. W. Partin, et al., "Obesity-Related Plasma Hemodilution and PSA Concentration among Men with Prostate Cancer," Journal of the American Medical Association, Vol. 298, No. 19, 2007, pp. 2275-2280. doi:10.1001/jama.298.19.2275

[21] R. L. Grubb, A. Black, G. Izmirlian, et al., "Serum Prostate-Specific Antigen Hemodilution among Obese Men Undergoing Screening in the Prostate, Lung, Colorectal, and Ovarian Cancer Screening Trial," Cancer Epidemiology, Biomarkers \& Prevention, Vol. 18, No. 3, 2009, pp. 748-751. doi:10.1158/1055-9965.EPI-08-0938

[22] S. Culp and M. Porter, "The Effect of Obesity and Lower Serum Prostate-Specific Antigen Levels on ProstateCancer Screening Results in American Men," British Journal of Urology International, Vol. 104, No. 10, 2009, pp. 1457-1461. doi:10.1111/j.1464-410X.2009.08646.x

[23] B. J. Davies, T. J. Walsh, P. L. Ross, et al., "Effect of BMI on Primary Treatment of Prostate Cancer," Urology, Vol. 72, No. 2, 2008, pp. 406-411. doi:10.1016/j.urology.2007.11.032

[24] J. Jayachandran, W. J. Aronson, M. K. Terris, et al., "Obesity and Positive Surgical Margins by Anatomic Location after Radical Prostatectomy: Results from the Shared Equal Access Regional Cancer Hospital Database," British Journal of Urology International, Vol. 102, No. 8, 2008, pp. 964-968. 
doi:10.1111/j.1464-410X.2008.07881.X

[25] J. S. Kasper, Y. Liu, M. N. Pollak, et al., "Hormonal Profile of Diabetic Men and the Potential Link to Prostate Cancer," Cancer Causes Control, Vol. 19, No. 7, 2008, pp. 703-710. doi:10.1007/s10552-008-9133-x

[26] I. Ben Sahra, K. Laurent, S. Giuliano, et al., "Targeting Cancer Cell Metabolism: The Combination of Metformin and 2-Deoxyglucose Induces p53-Dependent Apoptosis in Prostate Cancer Cells," Cancer Research, Vol. 70, No. 6, 2010, pp. 2465-2475.
doi:10.1158/0008-5472.CAN-09-2782

[27] A. Decensi, M. Puntoni, P. Goodwin, et al., "Metformin and Cancer Risk in Diabetic Patients: A Systematic Review and Meta-Analysis," Cancer Prevention Research, Vol. 3, No. 11, 2010, pp. 1451-1461. doi:10.1158/1940-6207.CAPR-10-0157

[28] C. J. Currie, C. D. Poole and E. A. Gale, "The Influence of Glucose-Lowering Therapies on Cancer Risk in Type 2 Diabetes," Diabetologia, Vol. 52, No. 9, 2009, pp. 17661777. doi:10.1007/s00125-009-1440-6 